\title{
New Applications of Nanotechnology for Neuroimaging
}

\author{
G. Suffredini, J.E. East, and L.M. Levy
}

\section{ABSTRACT}

SUMMARY: Advances in nanotechnology have the potential to dramatically enhance the detection of neurologic diseases with targeted contrast agents and to facilitate the delivery of focused therapies to the central nervous system. We present the physicochemical rationale for their use, applications in animal models, and ongoing clinical trials using these approaches. We highlight advances in the use of nanoparticles applied to brain tumor imaging, tumor angiogenesis, neurodegeneration, grafted stem cells, and neuroprogenitor cells.

ABBREVIATIONS: amyloid beta $=\mathrm{A} \beta ; \mathrm{NO}=$ nitric oxide; $\mathrm{NOS}=$ nitric oxide synthase; $\mathrm{PBCA}=$ poly $(n$-butyl cyanoacrylate); $\mathrm{QDots}=$ quantum dots; $\mathrm{SPIO}=$ superparamagnetic iron oxide

$\mathbf{R}$ ecent developments in nanotechnology have important implications for central nervous system imaging. These include pre- and intraoperative tumor imaging to facilitate accurate tumor characterization and resection, imaging of therapeutic stem cell delivery and viability in vivo, differentiation of pseudoprogression and pseudoresponse in antiangiogenic therapy for glioblastoma multiforme, detection of early stages of neurodegeneration, and advances in immunoimaging of the central nervous system. In this review, we will describe animal models, current and ongoing clinical trials, as well as future directions and implications for this exciting field.

\section{GOLD NANOCAGES AND PHOTOACOUSTIC IMAGING}

Gold nanocages are a novel class of optically tunable nanoparticles developed in the past decade. ${ }^{1}$ These nanoparticles are highly porous, hollow, cube-shaped structures with edge length measurements between 30 and $200 \mathrm{~nm} .^{2}$ Their surface plasmon resonance peaks can be tuned to have maximal optical absorption in the near-infrared spectrum. This spectrum is often referred to as the optical window of biologic tissues because in this spectrum, light attenuation by soft tissue and blood is low. ${ }^{3}$ Gold nanoparticles have been actively investigated for their role as optical and photoacoustic imaging contrast agents. ${ }^{4-7}$ The synthesis of gold

From the George Washington University School of Medicine and Health Sciences (G.S.), Washington, DC; Howard University School of Medicine (J.E.E.), Washington, DC; and Department of Radiology (L.M.L.), George Washington University Medical Center, Washington, DC

Please address correspondence to Lucien M. Levy, MD, PhD, Professor of Radiology and Director of Neuroradiology, George Washington University Medical Center, 900 23rd St, NW, Washington, DC 20037; e-mail: llevy@mfa.gwu.edu

- Indicates open access to non-subscribers at www.ajnr.org

http://dx.doi.org/10.3174/ajnr.A3543 nanocages and control of their surface plasmon resonance peaks has been reviewed. ${ }^{8}$

Photoacoustic tomography imaging is a hybrid technique that combines optical and sonographic imaging modalities and detects absorbed photons sonographically. This technique is based on the photoacoustic effect, in which energy absorption in the form of electromagnetic waves (optical and radio-frequency waves) can generate transient acoustic signals in a medium. ${ }^{7} \mathrm{Op}-$ tical and radio-frequency waves are the preferred waveforms used in photoacoustic imaging because they have more favorable properties, including deeper tissue penetration and higher absorption by contrast agents compared with other wave forms. ${ }^{7}$ More important, photoacoustic tomography provides spatial-resolution imaging at depths 50 times greater than optical imaging alone. ${ }^{2}$ Contrast agents such as gold nanocages can be tuned to have enhanced absorptive properties in the near-infrared region, which make them well-suited for photoacoustic imaging. ${ }^{5,9}$

Effective systemic delivery of nanoparticle contrast agents to interstitial tumor tissue relies on passive targeting through the enhanced permeability and retention effect. ${ }^{10}$ Unique features of tumors that contribute to this passive targeting phenomenon are an increased microattenuation of vessels with widened gap junctions and deranged lymphatic drainage. ${ }^{10}$ Intracellular delivery of nanoparticles can be achieved by linking to their surface antibodies or ligands (ie, albumin, transferrin, folate, lipoproteins, mannose, and others) to enable internalization by receptor-mediated endocytosis of the nanoparticle by specific targeted cells. ${ }^{11}$

Hollow gold nanospheres are effective photoacoustic contrast agents with the ability to depict brain blood vessels as small as 100 um in diameter. ${ }^{5}$ Following a single dose in a mouse model, the nanospheres had distribution half-lives near 
1.5 hours and elimination half-lives of nearly 71 hours and no evidence of systemic toxicity.

A novel application of photoacoustic imaging using gold nanocage contrast enhancement is accurate $3 \mathrm{D}$ imaging of brain tumors. Currently, a major limitation to gadolinium-based contrast imaging of brain tumors is the inaccurate margins encountered intraoperatively due to the disruption of the blood-brain barrier by surgery. Trimodal imaging by using MR imaging, Raman, and photoacoustic imaging by gold nanocage contrast enhancement provided highly accurate tumor description in a murine model, including delineation of the tumor parenchyma interface and microscopic tumor foci pre- and intraoperatively. ${ }^{6}$

These trimodal nanoparticles are composed of a 60-nm gold core, directly covered by a Raman active layer (trans-1.2[4-pyridyl]-ethylene). A 30-nm silica coating covers the Raman layer as a protective layer and maleimide-gadolinium-tetra-azacyclododecanetetra-acetic acid is linked to the silica layer. These nanoparticles accumulate in tumor via the enhanced permeability and retention effect preoperatively and are available as contrast for hours after the initial injection because of their long half-life. Because the contrast accumulates preferentially in tumor tissue and does not redistribute during surgical disruption of the bloodbrain barrier, these contrast agents allow more precise tumor resection than resection with gadolinium-based contrast agents. ${ }^{6}$

\section{EVALUATION AND VALIDATION OF ANTIANGIOGENIC THERAPY}

Antiangiogenic therapy has broad applications in cancer therapy, and the angiogenesis inhibitor bevacizumab has been used in the treatment of recurrent glioblastoma. ${ }^{12,13}$ Angiogenesis imaging is important to identify patients who would benefit from antiangiogenic therapies and to monitor treatment response. To date, there have been no well-validated radiologic markers to serve these purposes. Current assessment of tumor response via MR imaging is complicated by the "pseudoresponse" phenomenon, in which the treatment decreases the permeability of blood vessels giving the radiologic appearance of decreased enhancement on T1-weighted images, which may not reflect a true tumor response. ${ }^{14,15}$

Ferumoxytol is a superparamagnetic iron oxide (SPIO) nanoparticle that is approved for iron replacement therapy in patients with chronic kidney disease. ${ }^{16}$ Ferumoxytol acts as a "blood pool" agent, indicating that it is confined to the vascular compartment for hours after administration and will not extravasate in the presence of a compromised blood-brain barrier. Ferumoxytol was recently investigated for its potential superiority to gadolinium to distinguish pseudoprogression from true tumor progression in patients with glioblastoma multiforme by comparing relative cerebral blood volume estimated from dynamic susceptibilityweighted contrast-enhanced MR imaging. Ferumoxytol was significantly more sensitive in detecting tumor progression and differentiating pseudoprogression compared with gadolinium (Fig 1). ${ }^{17}$ There are several clinical trials currently evaluating the efficacy of ferumoxytol as an MR imaging contrast agent in evaluating brain malignancies (Table).

Other markers for targeted contrast agents include endothelial surface adhesion molecules whose over-expression is associated with vascular proliferation in tumor growth. The $\alpha_{\mathrm{v}} \beta_{3}$ integrin is associated with neovascular proliferation and has been used as a target to monitor angiogenesis. ${ }^{18-21}$ Optical imaging in the nearinfrared window $(650-900 \mathrm{~nm})$ with a functionalized cyclic fluorophore was recently shown to target overexpressed integrin receptors, enabling near-infrared fluorescent imaging of tumor tissue and tumor margins in both murine and human glioblastoma models. ${ }^{22}$ Because low-grade gliomas also express low levels of the $\alpha_{\mathrm{v}} \beta_{3}$ integrin receptor, this functionalized cyclic fluorophore also has the potential to image low-grade gliomas. ${ }^{23} \mathrm{Cur}-$ rently, a clinical trial in the recruitment phase is investigating the use of a functionalized cyclic fluorophore (IRDye800CW) conjugated to bevacizumab to determine its uptake and localization in breast tissue, surrounding healthy tissue, tumor margins, and lymph nodes. ${ }^{24}$

\section{DETECTION OF NITRIC OXIDE}

Nitric oxide is an inorganic gaseous molecule that is produced from L-arginine by nitric oxide (NO) synthase. NO has a number of physiologic functions related to neurotransmission and neovascularization and may contribute to neurotoxicity associated with tissue injury, apoptosis, and ischemia. ${ }^{25,26}$ It is important in the generation of nitrotyrosine, which is implicated in the progression of Alzheimer disease and Parkinson disease. ${ }^{27}$ As such, the detection of NO in biologic systems is an important area of research.

Classic methods used for detecting nitric oxide include spectroscopic and fluorescent dyes that rely on the reaction of $\mathrm{NO}$ with other molecules. ${ }^{28}$ A central limitation of these detection methods is their inability to measure NO concentrations in 3D. Multidimensional in vivo NO monitoring has been achieved by using amperometric trimethylsilane conjugated platinum nanodisks. ${ }^{29,30}$ This nanoscale sensor method was able to detect NOproducing neurons in a mouse model and was validated by immunohistochemistry (Fig 2). Although this method requires long acquisition times ( $\sim 100$ minutes $)$ and is limited to surface scanning, it has the potential to correlate NO production and disease states for diagnostic purposes in humans.

Positron-emission tomography imaging of endotoxin-induced nitric oxide synthase activation in the lungs of healthy volunteers is currently under investigation. ${ }^{31}$ In this investigation $\left[{ }^{18} \mathrm{~F}\right]( \pm)$ nitric oxide synthase (NOS) is the radioactive drug that targets inducible nitric oxide synthase. Alternative investigations may seek to target the delivery of radioactive imaging agents such as $\left[{ }^{18} \mathrm{~F}\right]( \pm)$ NOS to the CNS in patients who have Alzheimer disease, Parkinson disease, and other degenerative neurologic conditions in which nitric oxide is thought to play a central role in neural toxicity. Such an experiment could involve the delivery of agents such as $\left[{ }^{18} \mathrm{~F}\right]( \pm)$ NOS in a vehicle such as poly $(n$-butyl cyanoacrylate) (PBCA) nanoparticles coated with the surfactant polysorbate $80 \mathrm{PBCA}$ that would bypass the blood-brain barrier.

\section{STEM CELL TRACKING WITH MR IMAGING}

Regenerative therapy by using adult neuronal stem cells may have therapeutic potential in neurodegenerative, ${ }^{32}$ ischemic, ${ }^{33}$ and traumatic ${ }^{34}$ brain injuries as well as systemic diseases such as diabetes. ${ }^{35}$ Adult neurogenesis is restricted to the subgranular zone of the hippocampal dentate gyrus and to the subventricular zone 
No contrast

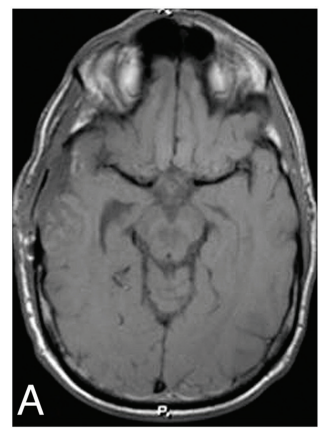

\section{Before RCT}

\section{After RCT}

\section{4 months after RCT}

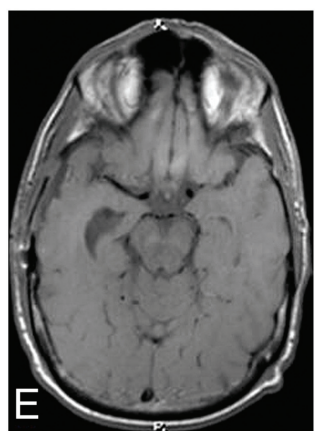

$\mathrm{B}$
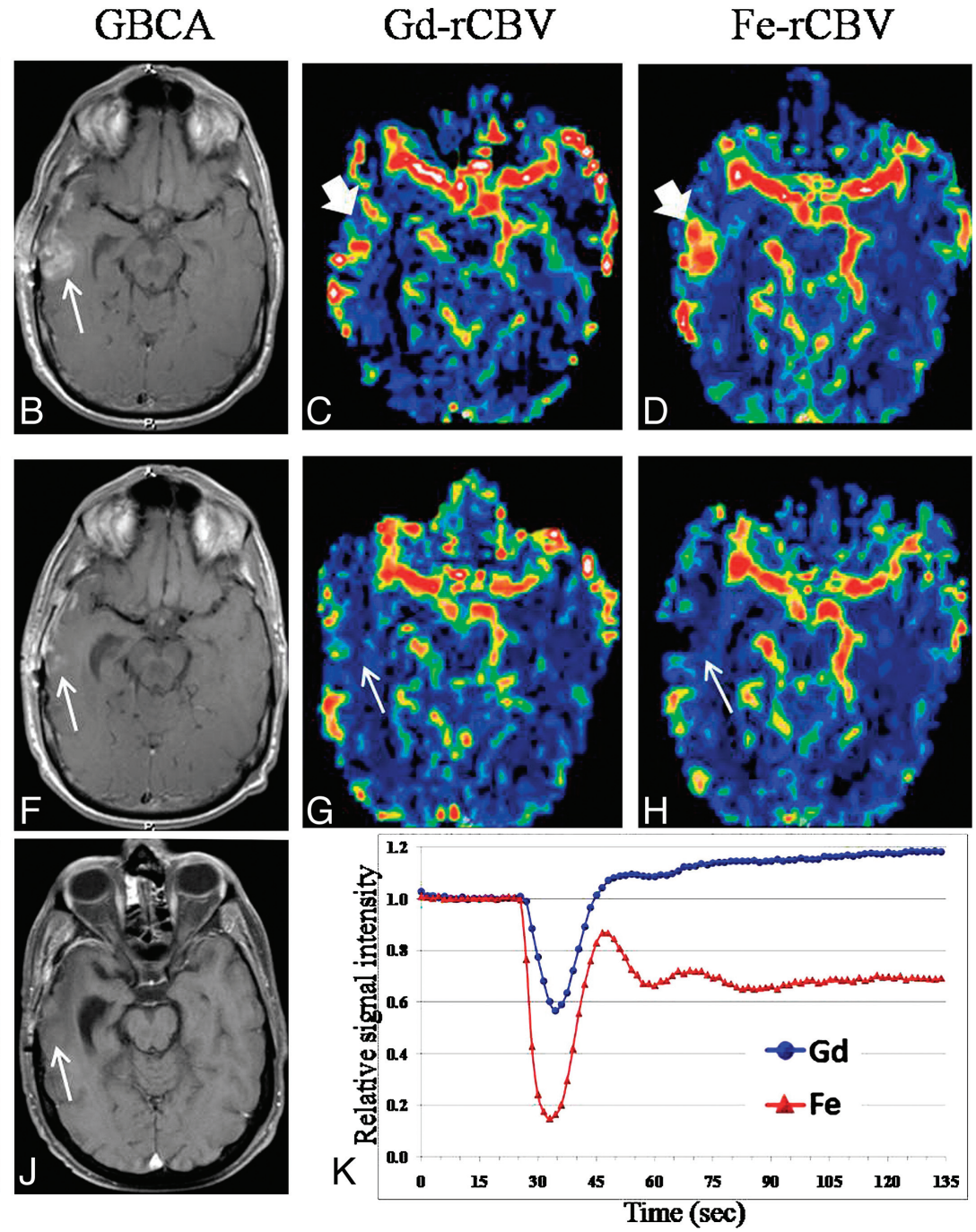

FIG 1. Dynamic susceptibility contrast MR imaging comparing gadoteridol and the iron oxide nanoparticle ferumoxytol in the evaluation of treatment response to standard radiochemotherapy for glioblastoma multiforme. RCT indicates regression after radiochemotherapy. T1weighted MR imaging before $(A, E$, and $I)$ and after $(B, F$, and $/)$ gadoteridol (Gd) administration. $A$ and $B$, MR imaging after surgery but before RCT demonstrates an area of enhancement in the right temporal lobe (arrow). Relative cerebral blood volume (rCBV) in the enhancing area was lower on the Gd-rCBV parametric map (C) compared with the ferumoxytol (Fe)-rCBV map (D) (bold arrow). E and $F$, MR imaging after completion of RCT revealed decreased enhancement $(F$, arrow) with low $r C B V$ on $G d-r C B V(G)$ and Fe-rCBV parametric maps $(H)$ (arrow). I and $)$, MR imaging 14 months after completion of RCT showed resolution of enhancement. $K$, First-pass time-intensity curves of the perfusions in $C$ and $D$ demonstrate postbolus increasing signal above the baseline when $\mathrm{Gd}$ was used, while ferumoxytol postbolus signal intensity is below the baseline and remains stable. Reprinted from IntJ Radiat Oncol Biol Phys, Volume 79(2):514-23, Gahramanov S, Raslan AM, Muldoon LL. Potential for Differentiation of Pseudoprogression From True Tumor Progression With Dynamic Susceptibility-Weighted Contrast-Enhanced Magnetic Resonance Imaging Using Ferumoxytol vs. Gadoteridol: A Pilot Study. Copyright 2011, with permission from Elsevier.

adjacent to the lateral ventricles. ${ }^{36}$ Current methods for neuronal regeneration include the use of scaffolds, ${ }^{37}$ transcription factors, ${ }^{38}$ or direct stem cell implantation. ${ }^{39}$ Noninvasive in vivo imaging of these stem cell-based therapies is important to guide treatment as well as to evaluate the therapeutic effect in animal models and, eventually, patients.

MR imaging is well-suited for this goal and has been shown to be safe and effective in clinical trials tracking cells labeled with superparamagnetic iron oxide nanoparticles. ${ }^{40}$ The SPIO nanoparticles are biocompatible and are degraded through the normal iron metabolism mechanisms in the body. ${ }^{41}$ Clinical trials based on this approach include labeling and tracking of dendritic cells in immunotherapy of melanoma, autologous neural stem cells in traumatic head injury, bone marrow stem cells in chronic spinal cord injury, and cadaveric islet cells infused intraportally in the liver. $^{40}$

The utility of SPIO nanoparticles for long-term in vivo cell tracking was investigated in allogeneic neural stem cell transplants performed in wild type (graft rejecting) BALb/c and immunodeficient (graft accepting) Rag2 mice. ${ }^{42}$ SPIO-loaded neural stem cells were implanted in the corpus callosum of both sets of mice, and the progenitor cells were allowed to grow for 95 days while being monitored by MR imaging, immunohistochemistry, and bioluminescence.

The rate of MR signal decline defined 2 populations of cells. The early disappearance of the MR signal was associated with cell proliferation, thus diluting the amount of contrast per cell. The prolongation of the MR signal was associated with cell death and 


\begin{tabular}{|c|c|c|}
\hline Study Title & Phase & Clinical Trial Description \\
\hline $\begin{array}{l}\text { Imaging vascular properties of pediatric brain } \\
\text { tumors using ferumoxytol and gadolinium in a } \\
\text { single imaging session; an } \mathrm{NCl} \text {-sponsored } \\
\text { exploratory trial (code } 7228 \text { ) (NCT00978562) }\end{array}$ & 0 & $\begin{array}{l}\text { To assess the safety and effectiveness of } \\
\text { ferumoxytol in improving the ability to image } \\
\text { pediatric brain tumors }\end{array}$ \\
\hline $\begin{array}{l}\text { MRI using ferumoxytol in patients with primary } \\
\text { brain cancer or brain metastases from lung or } \\
\text { breast cancer; an } \mathrm{NCl} \text {-sponsored } \\
\text { multidisciplinary study (NCT00103038) }\end{array}$ & 2 & $\begin{array}{l}\text { Characterization of vascular properties of tumors } \\
\text { in the CNS using ferumoxytol for dynamic- } \\
\text { susceptibility contrast MRI to compare with } \\
\text { those obtained using a gadolinium-based } \\
\text { contrast agent for dynamic contrast-enhanced } \\
\text { imaging in a single MR imaging session; imaging } \\
\text { properties will be assessed longitudinally with up } \\
\text { to } 6 \text { imaging sessions for } 2 \text { years }\end{array}$ \\
\hline $\begin{array}{l}\text { Magnetic resonance (MR) imaging study using ferumoxytol to assess } \\
\text { early tumor response in patients with } \\
\text { glioblastoma multiforme (code 7228) } \\
\text { (NCT00660543) }\end{array}$ & 1 & $\begin{array}{l}\text { Evaluating the ability of ferumoxytol to assess } \\
\text { effective early treatment response in } \\
\text { glioblastoma multiforme using dynamic } \\
\text { perfusion, BBB permeability measurement }\end{array}$ \\
\hline $\begin{array}{l}\text { Ferumoxytol and gadolinium magnetic resonance } \\
\text { imaging (MRI) at 3T and 7T in patients with } \\
\text { malignant brain tumors (NCT00659126) }\end{array}$ & 2 & $\begin{array}{l}\text { Comparing dynamic perfusion, BBB permeability } \\
\text { measurement in } 2 \text { different magnetic fields } \\
\text { ( } 3 \mathrm{~T} \text { and } 7 \mathrm{~T} \text { ) in the evaluation of brain tumors; } \\
\text { gadolinium and ferumoxytol will be used as } \\
\text { contrast agents }\end{array}$ \\
\hline $\begin{array}{l}\text { Assessing dynamic magnetic resonance (MR) imaging in patients with } \\
\text { recurrent high grade glioma receiving } \\
\text { chemotherapy (code 7228) (NCT00769093) }\end{array}$ & 1 & $\begin{array}{l}\text { Evaluation of imaging changes induced by } \\
\text { bevacizumab with dexamethasone in patients } \\
\text { with high-grade glioma }\end{array}$ \\
\hline $\begin{array}{l}\text { MR, histologic and EM imaging of intravenous } \\
\text { ferumoxytol in central nervous system (CNS) } \\
\text { inflammation (NCT00659776) }\end{array}$ & 2 & $\begin{array}{l}\text { Evaluation of the safety and efficacy of } \\
\text { ferumoxytol }\end{array}$ \\
\hline
\end{tabular}

phagocytic contrast retention. The authors of the study note that this finding was unexpected and may be related to the cell line used or the method of stem cell implantation (eg, direct implantation into the parenchyma or via the CSF). Thus, although shortterm in vivo SPIO-labeled cell imaging is considered accurate, the long-term accuracy of SPIO-labeled cell imaging is complex and the authors of this study suggested that it be used only if the viability of the implanted cells is known.

A clinical trial pilot study is currently under investigation to assess the utility of MR imaging in tracking intravenously delivered SPIO-labeled stem cells in healthy human volunteers for periods of up to 1 week. ${ }^{43}$

\section{NONINVASIVE DETECTION OF NEURAL PROGENITOR CELLS IN LIVING BRAINS BY MR IMAGING}

Pericytes are a source of adult multipotent progenitor cells ${ }^{44}$ located in the central nervous system between the inner and outer vascular basement membrane of CNS capillaries, and they function in the regulation of brain capillary blood flow, angiogenesis, and blood-brain barrier maintenance. ${ }^{45-47}$ Recently, noninvasive imaging of neural progenitor cells during angiogenesis was demonstrated in a bilateral carotid artery occlusion murine model by using gene transcript-targeted MR imaging contrast agents. In this ischemic brain injury model, SPIO nanoparticles, modified with micro DNA targeting actin and nestin messenger ribonucleic acid (the latter uniquely expressed by pericytes), were administered to mice after bilateral carotid artery occlusion to image vessel formation by the detection of cerebral pericytes. ${ }^{48}$ The dual gene transcript-targeted MR imaging successfully identified pericytes in living brains. Angiogenesis is dysregulated in several disease states including Alzheimer disease ${ }^{49}$ and diabetes mellitus, ${ }^{50}$ and the ability to image CNS pericyte activity in vivo may allow real-time longitudinal monitoring of angiogenesis in these disease states.

\section{ENHANCED BRAIN DELIVERY OF BLOOD-BRAIN BARRIER IMPERMEABLE PROBES FOR OPTICAL AND MR IMAGING}

Polymeric nanoparticles describe both nanospheres and nanocapsules. These are sphere-like structures composed of biodegradable polymers, and they contain a payload delivery confined to a central cavity ${ }^{51}$ or dispersed within a matrix, respectively (Fig 3). ${ }^{52}$ Nanoparticles composed of poly( $n$-butyl cyanoacrylate) coated with the surfactant polysorbate 80 can bypass the BBB by binding to apolipoprotein $E$ followed by adhesion and receptormediated transcytosis via the low-attenuation lipoprotein receptor present on BBB endothelial cells. ${ }^{53,54}$ This phenomenon has the potential to allow in vivo molecular imaging. Studies describing nanocarriers traversing the BBB have, in large part, relied on postmortem analysis; however, recently the delivery of BBB impermeable molecular imaging contrast agents for in vivo microscopy and MR imaging has been performed. ${ }^{55}$ In this study, the delivery of a series of compounds known not to cross the BBB in their native state, including a DNA-binding dye bisBenzimide used to monitor neuronal nuclei; Texas red dextran, a targeted probe for imaging lesions characteristic of Alzheimer disease; and tryptan blue, a plaque-binding red fluorescing diazo dye, were delivered intravenously as 2 formulations: native species and encapsulated in polysorbate 80-coated PBCA nanoparticles. Each compound was found to traverse the $\mathrm{BBB}$ when encapsulated in polysorbate 80 - coated PBCA nanoparticles and was found not to traverse the $\mathrm{BBB}$ when delivered in native form. Furthermore, the delivery of gadolinium-based MR imaging contrast agents 

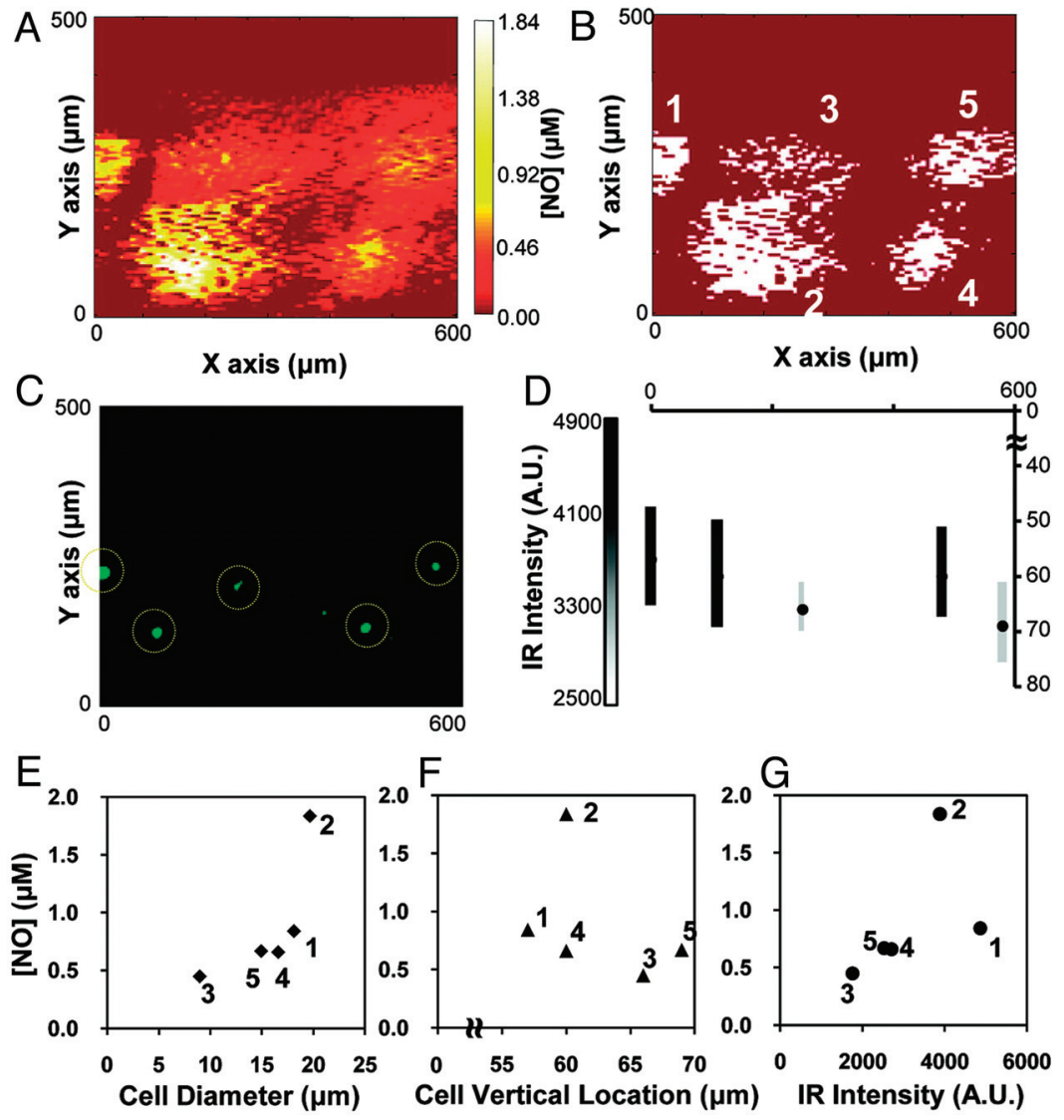

FIG 2. Real-time in vivo nitric oxide imaging with corresponding immunohistochemical analysis of neuronal NO synthase immunoreactive (nNOS-IR) cells. In vivo NO measurements and immunohistochemical analysis of nNOS-IR cells. A, A NO concentration image acquired over the cortical surface of a $600 \times 500 \mu \mathrm{m}^{2}$ region. Higher NO concentrations are shown in lighter color, whereas lower NO concentrations are shown as a darker red. B, A thresholded image of $A$. The highest $10 \% \mathrm{NO}$ concentrations of the entire concentration range are illustrated in white, whereas the other lower concentrations are in red. There are 5 distinct sections with high NO concentrations (labeled 1-5). C, A confocal microscopy image of nNOS-IR cells. The locations of yellowcircled cells are well-matched with the sites exhibiting relatively high NO concentrations in A. D, Five distinct nNOS-IR cells are plotted on the basis of the location information in the $x$ - and $\mathrm{z}$-axes. Each gray-scaled bar represents a corresponding single nNOS-IR cell. The color of each bar reflects the intensity of immunoreactivity (ie, darker color indicates strong immunoreactivity). The highest NO concentration measured in each section (labeled 1-5) is plotted as a function of $E$, the corresponding nNOS- IR cell size; the vertical cell location (depth) (F); and the immunoreactivity intensity of the cell $(G)$ in the confocal image. $E-G$, The NO concentration means the highest NO level within each localized site. The NOS-IR cell size means the largest diameter of each cell observed in the confocal image. The cell location in depth means the vertical distance from the cortical upper surface to the cell center position. The inversion recovery intensity means the highest fluorescence intensity measured by confocal microscopy within each cell. Reprinted with permission from Jo A, Do H, Jhon GJ, et al. Electrochemical nanosensor for real-time direct imaging of nitric oxide in living brain. Anal Chem 83:8314-19. Copyright 2011, American Chemical Society.

encapsulated in polysorbate 80 -coated PBCA nanoparticles enhanced brain delivery of the contrast agent several hundredfold compared with delivery of free gadolinium, indicating the future possibility of targeted MR imaging contrast agents into the brain.

\section{NANOBODIES}

Nanobodies are a class of antibodies initially discovered in serum of the camel species Camelus dromedarius. They consist of heavy chains only and have desirable features such as small size, decreased immunogenicity, and high binding affinities. ${ }^{56}$ Nanobodies have been designed for use in cancer therapy, ${ }^{57,58}$ antivenoms, ${ }^{59}$ inflammatory conditions, ${ }^{60}$ and immunoimaging. ${ }^{60-64}$
Nanobodies can detect vascular and parenchymal amyloid beta $(\mathrm{A} \beta)$ deposits in vivo to differentiate cerebral $\beta$-amyloid indicative of Alzheimer disease and vascular $A \beta$ plaques associated with cerebral amyloid angiopathy. ${ }^{65}$

With the transgenic Alzheimer Disease Cerebral Amyloid Angiopathy (AD/CAA) mouse model, 2 distinct $\mathrm{A} \beta$ targeting nanobodies, ni3A and $\mathrm{pa} 2 \mathrm{H}$, were administered to evaluate their ability in vivo to differentially detect vascular or parenchymal amyloid- $\beta$ deposits and their ability to cross the blood-brain barrier. In vivo specificity for $A \beta$ was evaluated in this mouse model by direct topical application or intracarotid coinjection with mannitol with a fluorescently labeled nanobody. The in vivo murine models showed that both ni3A and pa2H had affinity for parenchymal and vascular deposits. However, in vitro topical applications to human brain tissue of patients with $\mathrm{AD} / \mathrm{CAA}$ showed ni3A to specifically target only vascular $\mathrm{A} \beta$.

Although there are specific active transport mechanisms known to be involved in the transport of nanobodies across the blood-brain barrier, ${ }^{66}$ the nanobodies in this study were observed to have a limited ability to cross the BBB. Limited in vivo BBB passage was postulated by the authors to be due to low concentrations of nanobodies, low circulation time, or possibly a paucity of active transport receptors involved in BBB transport in this mouse model. ${ }^{65}$ Future methods, such as nanobodies encapsulated in polysorbate 80 -coated PBCA nanoparticles, could be used to increase in vivo BBB passage.

\section{QUANTUM DOTS}

Quantum dots (QDots) are 10- to 20-nm nanoparticles that consist of semiconductor material surrounded by a polymer shell. The polymer shell can then be functionalized; this process allows the linking of any number of biologically relevant molecules (oligopeptides, antibodies, and so forth) to the surface. These functionalized quantum dots have had applications in cellular imaging, ${ }^{67,68}$ protein detection, ${ }^{69}$ and cancer immunology. ${ }^{70}$ Because of their unique charge-carrying abilities, QDots possess molar extinction coefficients 10-50 times greater than typical organic dyes and are also up to 20 times brighter. ${ }^{71}$ Additionally QDots have decreased rates of photobleaching, which lends them to being well-suited for biologic imaging.

By varying the semiconductor material contained within quantum dot nanoparticles, one can manipulate the wavelength 


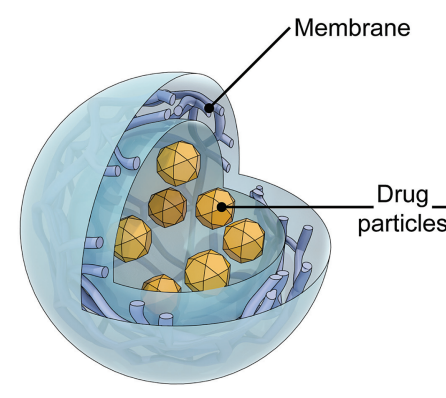

Nanocapsule

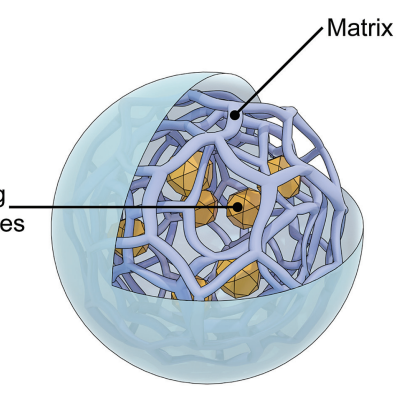

Nanosphere
FIG 3. Schematic representation of the structure of a nanocapsule and a nanosphere. This research was supported by the Intramural Research Program of the National Institute of Neurologic Disorders and Stroke at the National Institutes of Health. The image is in the public domain and credited to the National Institutes of Health/Department of Health and Human Services.

that is emitted on excitation to skew emissions to the near-infrared portion of the electromagnetic spectrum. This region is optimal for deep-tissue imaging due to the optical characteristics of biologic tissue. ${ }^{3}$

Recently, a quantum dot fluorescent probe designed to target the epidermal growth factor receptor was evaluated for its ability to differentiate tumor cells from normal brain parenchyma in tumor biopsies of low-grade glioma. ${ }^{72}$ In this study, quantum dots were conjugated to epidermal growth factor as well as monoclonal antibodies directed against the epidermal growth factor receptor and were evaluated for their ability to differentiate normal brain parenchyma from human glioma ex vivo (Fig 4). Tumor cells could be visualized from the macroscopic level, and this result indicated that quantum dot-targeted fluorescent probes could be used intraoperatively to assist in discerning tumor tissue from normal parenchyma in the resection of low-grade gliomas.

\section{CONCLUSIONS}

Nanotechnology is an exciting and burgeoning field that will greatly impact medical diagnostics and therapeutics across many medical specialties in the coming age of personalized and targeted medicine. These impacts will likely be seen in areas of stem cell therapy, immunoimaging and immunotherapy, enhanced preand intraoperative brain tumor characterization, and targeted payload delivery to the CNS.

The clinical development of neural stem cell therapies will require accurate tracking of their in vivo location and viability. SPIOs appear well-suited for this need. The delivery and monitoring of therapeutic stem cells is likely to play an important role for interventional and diagnostic radiologists in the treatment of autoimmune and degenerative diseases, including diabetes, multiple sclerosis, Parkinson disease, Alzheimer disease, amyotrophic lateral sclerosis, and spinal muscle atrophy.

Nanobodies are less immunogenic than monoclonal antibodies, are easy to produce, and can cross the BBB by their own unique mechanisms, opening up numerous opportunities for immunoimaging of the CNS and expanding the role of radiologists in molecular-based diagnostics of the CNS.

The pre- and intraoperative imaging of brain tumors with multifunctional nanoparticles combining photoacoustic, Raman, and MR imaging and/or QDots may facilitate more accurate tumor characterization and resection. Furthermore, ferumoxytol, a blood pool agent, has shown great potential in distinguishing pseudoprogression and pseudoresponse from true tumor progression and true treatment response in glioblastoma.
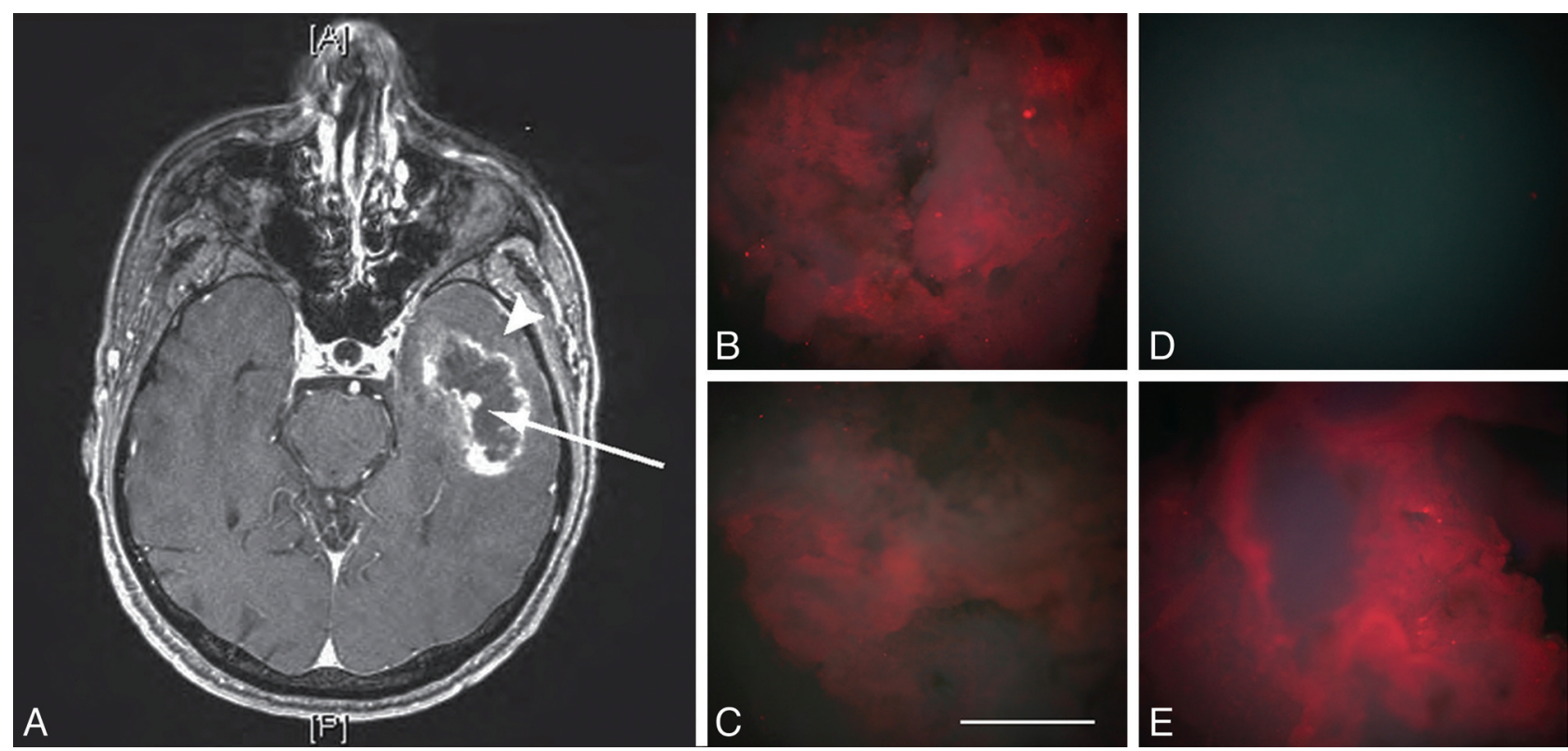

FIG 4. $M R$ imaging and quantum dot (QD)-probe digital macroimages from glioblastoma multiforme, grade IV biopsy. Example of QD targeted to epidermal growth factor receptor (EGFR) to discriminate tumor tissue from normal parenchyma at the margin of a resected glioblastoma multiforme. A, T1-weighted MR axial image shows gadolinium-positive signal. $B-E$, Digital macrophotographic images of ex vivo stained biopsies from the resected tumor and adjacent brain tissue stained with targeted QD probes obtained with the same magnification and the same exposure times. B, Tumor tissue 625QDStAv-biotin-MAb528 EGFR staining. C, 625QDGAMIG-MAb 199.12 EGFR staining. D, Adjacent brain 625QDStAv-biotin-MAb528 EGFR staining. E, Invading tumor tissue, 625QDStAv-biotin-MAb528 EGFR staining. Excitation, $365 \mathrm{~nm}$; emission, $>450 \mathrm{~mm}$; objective, $5 \mathrm{X}$ numeric aperture 0.15 ; bar $1 \mathrm{~mm}$. Note that $D$ serves as the control for $B$ (ie, stained with the same probes under identical conditions). (Copyright $\odot 2010$ Kantelhardt al. ${ }^{72}$ ) 
The ability to accurately bypass the BBB with PBCA nanoparticles coated with polysorbate 80 and to deliver a payload such as a contrast agent or drug is no doubt an important and unique feature of nanobased applications that yields myriad possibilities for future treatment and imaging of the CNS.

\section{REFERENCES}

1. Sun Y, Xia Y. Shape-controlled synthesis of gold and silver nanoparticles. Science 2002;298:2176-79

2. Li W, Brown PK, Wang LV, et al. Gold nanocages as contrast agents for photoacoustic imaging. Contrast Media Mol Imaging 2011;6:370-77

3. Weissleder R. A clearer vision for in vivo imaging. Nat Biotechnol 2001;19:316-17

4. Chen J, Saeki F, Wiley BJ, et al. Gold nanocages: bioconjugation and their potential use as optical imaging contrast agents. Nano Lett 2005;5:473-77

5. Lu W, Huang Q, Ku G, et al. Photoacoustic imaging of living mouse brain vasculature using hollow gold nanospheres. Biomaterials 2010;31:2617-26

6. Kircher MF, de la Zerda A, Jokerst JV, et al. A brain tumor molecular imaging strategy using a new triple-modality MRI-photoacousticRaman nanoparticle. Nat Med 2012;18:829-34

7. Zhang Y, Hong H, Cai W. Photoacoustic imaging. Cold Spring Harb Protoc 2011:pbd.top065508

8. Hu M, Chen J, Li ZY, et al. Gold nanostructures: engineering their plasmonic properties for biomedical applications. Chem Soc Rev 2006;35:1084-94

9. Yang X, Skrabalak SE, Li ZY, et al. Photoacoustic tomography of a rat cerebral cortex in vivo with au nanocages as an optical contrast agent. Nano Lett 2007;7:3798-802

10. Maeda H, Fang J, Inutsuka T, et al. Vascular permeability enhancement in solid tumor: various factors, mechanisms involved and its implications. Int Immunopharmacol 2003;3:319-28

11. Peer DK, Hong S, Farokhzad OC, et al. Nanocarriers as an emerging platform for cancer therapy. Nat Nanotechnol 2007;2:751-60

12. Gerstner ER, Batchelor TT. Antiangiogenic therapy for glioblastoma. Cancer J 2012;18:45-50

13. Sorensen AG, Emblem KE, Polaskova P, et al. Increased survival of glioblastoma patients who respond to antiangiogenic therapy with elevated blood perfusion. Cancer Res 2012;72:402-07

14. Clarke JL, Chang S. Pseudoprogression and pseudoresponse: challenges in brain tumor imaging. Curr Neurol Neurosci Rep 2009;9:241-46

15. Pope WB, Young JR, Ellingson BM. Advances in MRI assessment of gliomas and response to anti-VEGF therapy. Curr Neurol Neurosci Rep 2011;11:336-44

16. Lu M, Cohen MH, Rieves D, et al. FDA report: ferumoxytol for intravenous iron therapy in adult patients with chronic kidney disease. Am J Hematol 2010;85:315-19

17. Gahramanov S, Raslan AM, Muldoon LL, et al. Potential for differentiation of pseudoprogression from true tumor progression with dynamic susceptibility-weighted contrast-enhanced magnetic resonance imaging using ferumoxytol vs. gadoteridol: a pilot study. Int J Radiat Oncol Biol Phys 2011;79:514-23

18. Shattil SJ. Function and regulation of the beta 3 integrins in hemostasis and vascular biology. Thromb Haemost 1995;74:149-55

19. De S, Razorenova O, McCabe NP, et al. VEGF-integrin interplay controls tumor growth and vascularization. Proc Natl Acad Sci US A 2005;102:7589-94

20. Varner JA, Cheresh DA. Tumor angiogenesis and the role of vascular cell integrin alphavbeta3. Important Adv Oncol 1996;69-87

21. Folkman J. Angiogenesis in cancer, vascular, rheumatoid and other diseases. Nat Med 1995;1:27-31

22. Huang R, Vider J, Kovar JL, et al. Integrin alphavbeta3-targeted IRDye 800CW near-infrared imaging of glioblastoma. Clin Cancer Res 2012;18:5731-40
23. Schnell O, Krebs B, Wagner E, et al. Expression of integrin alphavbeta 3 in gliomas correlates with tumor grade and is not restricted to tumor vasculature. Brain Pathol 2008;18:378-86

24. Groningen UMC. VEGF-targeted Fluorescent Tracer Imaging in Breast Cancer. http://clinicaltrials.gov/ct2/show/record/NCT01508572. Accessed November 8, 2012

25. Vincent SR. Nitric oxide neurons and neurotransmission. Prog Neurobiol 2010;90:246-55

26. Calabrese V, Mancuso C, Calvani M, et al. Nitric oxide in the central nervous system: neuroprotection versus neurotoxicity. Nat Rev Neurosci 2007;8:766-75

27. Garthwaite J, Boulton CL. Nitric oxide signaling in the central nervous system. Annu Rev Physiol 1995;57:683-706

28. Hong H, Sun J, Cai W. Multimodality imaging of nitric oxide and nitric oxide synthases. Free Radic Biol Med 2009;47:684-98

29. Jo A, Do H, Jhon GJ, et al. Electrochemical nanosensor for real-time direct imaging of nitric oxide in living brain. Anal Chem 2011;83:8314-19

30. Shim JH, Lee Y. Amperometric nitric oxide microsensor based on nanopore-platinized platinum: the application for imaging NO concentrations. Anal Chem 2009;81:8571-76

31. Medicine WUSo. PET imaging of endotoxin-induced iNOS activation. http://clinicaltrials.gov/ct2/show/NCT01407796. Accessed November 18, 2012

32. Borlongan CV. Recent preclinical evidence advancing cell therapy for Alzheimer's disease. Exp Neurol 2012;237:142-46

33. Song M, Mohamad O, Gu X, et al. Restoration of intracortical and thalamocortical circuits after transplantation of bone marrow mesenchymal stem cells into the ischemic brain of mice. Cell Transplant 2013;22:2001-15

34. Ugoya SO, Tu J. Bench to bedside of neural stem cell in traumatic brain injury. Stem Cells Int 2012;2012:141624

35. Kuwabara T, Asashima M. Regenerative medicine using adult neural stem cells: the potential for diabetes therapy and other pharmaceutical applications. J Mol Cell Biol 2012;4:133-39

36. Gage FH, Ray J, Fisher LJ. Isolation, characterization, and use of stem cells from the CNS. Annu Rev Neurosci 1995;18:159-92

37. Migliorini E, Grenci G, Ban J, et al. Acceleration of neuronal precursors differentiation induced by substrate nanotopography. Biotechnol Bioeng 2011;108:2736-46

38. Liu H, Zhang SC. Specification of neuronal and glial subtypes from human pluripotent stem cells. Cell Mol Life Sci 2011;68:3995-4008

39. Schwarz SC, Schwarz J. Translation of stem cell therapy for neurological diseases. Transl Res 2010;156:155-60

40. Bulte JW. In vivo MRI cell tracking: clinical studies. AJR Am J Roentgenol 2009;193:314-25

41. Ferrucci JT, Stark DD. Iron oxide-enhanced MR imaging of the liver and spleen: review of the first 5 years. AJR Am J Roentgenol 1990;155:943-50

42. Berman SC, Galpoththawela C, Gilad AA, et al. Long-term MR cell tracking of neural stem cells grafted in immunocompetent versus immunodeficient mice reveals distinct differences in contrast between live and dead cells. Magn Reson Med 2011;65:564-74

43. University of Edinburgh. Cell tracking using superparamagnetic particles of iron oxide (SPIO) and magnetic resonance imaging (MRI): a pilot study. http://www.clinicaltrials.gov/ct2/show/NCT00972946. Accessed November 18, 2012

44. Dore-Duffy P, Katychev A, Wang X, et al. CNS microvascular pericytes exhibit multipotential stem cell activity. J Cereb Blood Flow Metab 2006;26:613-24

45. Rodriguez-Baeza A, Reina-De La Torre F, Ortega-Sanchez M, et al. Perivascular structures in corrosion casts of the human central nervous system: a confocal laser and scanning electron microscope study. Anat Rec 1998;252:176-84

46. Itoh Y, Suzuki N. Control of brain capillary blood flow. JCereb Blood Flow Metab 2012;32:1167-76

47. Bergers G, Song $\mathrm{S}$. The role of pericytes in blood-vessel formation and maintenance. Neuro Oncol 2005;7:452-64 
48. Liu CH, Ren JQ, You Z, et al. Noninvasive detection of neural progenitor cells in living brains by MRI. FASEB J 2012;26:1652-62

49. Kalaria RN, Cohen DL, Premkumar DR, et al. Vascular endothelial growth factor in Alzheimer's disease and experimental cerebral ischemia. Brain Res Mol Brain Res 1998;62:101-05

50. Zhu M, Bi X, Jia Q, et al. The possible mechanism for impaired angiogenesis after transient focal ischemia in type 2 diabetic GK rats: different expressions of angiostatin and vascular endothelial growth factor. Biomed Pharmacother 2010;64:208-13

51. Leary SP, Liu CY, Apuzzo ML. Toward the emergence of nanoneurosurgery. Part III. Nanomedicine: targeted nanotherapy, nanosurgery, and progress toward the realization of nanoneurosurgery. Neurosurgery 2006;58:1009-26, discussion 1026

52. Pinto Reis C, Neufeld RJ, Ribeiro AJ, et al. Nanoencapsulation I. Methods for preparation of drug-loaded polymeric nanoparticles. Nanomedicine 2006;2:8-21

53. Steiniger SC, Kreuter J, Khalansky AS, et al. Chemotherapy of glioblastoma in rats using doxorubicin-loaded nanoparticles. Int J Cancer 2004;109:759-67

54. Hasadsri L, Kreuter J, Hattori H, et al. Functional protein delivery into neurons using polymeric nanoparticles. $J$ Biol Chem 2009;284:6972-81

55. Koffie RM, Farrar CT, Saidi LJ, et al. Nanoparticles enhance brain delivery of blood-brain barrier-impermeable probes for in vivo optical and magnetic resonance imaging. Proc Natl Acad Sci U S A 2011;108:18837-42

56. Hamers-Casterman C, Atarhouch T, Muyldermans S, et al. Naturally occurring antibodies devoid of light chains. Nature 1993;363:446-48

57. Tijink BM, Laeremans $\mathrm{T}$, Budde M, et al. Improved tumor targeting of anti-epidermal growth factor receptor nanobodies through albumin binding: taking advantage of modular nanobody technology. Mol Cancer Ther 2008;7:2288-97

58. Roovers RC, Vosjan MJ, Laeremans T, et al. A biparatopic anti-EGFR nanobody efficiently inhibits solid tumour growth. Int $J$ Cancer 2011;129:2013-24

59. Hmila I, Saerens D, Ben Abderrazek R, et al. A bispecific nanobody to provide full protection against lethal scorpion envenoming. FASEB J 2010;24:3479-89

60. Coppieters K, Dreier T, Silence K, et al. Formatted anti-tumor necrosis factor alpha $\mathrm{VHH}$ proteins derived from camelids show superior potency and targeting to inflamed joints in a murine model of collagen-induced arthritis. Arthritis Rheum 2006;54:1856-66

61. Devoogdt N, Xavier C, Hernot S, et al. Molecular imaging using nanobodies: a case study. Methods Mol Biol 2012;911:559-67

62. Vaneycken I, D'Huyvetter M, Hernot S, et al. Immuno-imaging using nanobodies. Curr Opin Biotechnol 2011;22:877-81

63. Vaneycken I, Devoogdt N, Van Gassen N, et al. Preclinical screening of anti-HER2 nanobodies for molecular imaging of breast cancer. FASEB J 2011;25:2433-46

64. Schoonooghe S, Laoui D, Van Ginderachter JA, et al. Novel appli- cations of nanobodies for in vivo bio-imaging of inflamed tissues in inflammatory diseases and cancer. Immunobiology 2012;217:1266-72

65. Nabuurs RJ, Rutgers KS, Welling MM, et al. In vivo detection of amyloid-beta deposits using heavy chain antibody fragments in a transgenic mouse model for Alzheimer's disease. PLoS One 2012;7:e38284

66. Rutgers KS, Nabuurs RJ, van den Berg SA, et al. Transmigration of beta amyloid specific heavy chain antibody fragments across the in vitro blood-brain barrier. Neuroscience 2011;190:37-42

67. Pathak S, Choi SK, Arnheim N, et al. Hydroxylated quantum dots as luminescent probes for in situ hybridization. J Am Chem Soc 2001;123:4103-04

68. Bruchez M Jr, Moronne M, Gin P, et al. Semiconductor nanocrystals as fluorescent biological labels. Science 1998;281:2013-16

69. Sukhanova A, Devy J, Venteo L, et al. Biocompatible fluorescent nanocrystals for immunolabeling of membrane proteins and cells. Anal Biochem 2004;324:60-67

70. Sun W, Subrahmanyam PB, East JE, et al. Connecting the dots: artificial antigen presenting cell-mediated modulation of natural killer T cells. J Interferon Cytokine Res 2012;32:505-16

71. Smith AM, Dave S, Nie S, et al. Multicolor quantum dots for molecular diagnostics of cancer. Expert Rev Mol Diagn 2006;6:231-44

72. Kantelhardt SR, Caarls W, de Vries AH, et al. Specific visualization of glioma cells in living low-grade tumor tissue. PLoS One 2010;5:e11323

73. OHSU Knight Cancer Institute. Imaging vascular properties of pediatric brain tumors using ferumoxytol and gadolinium in a single imaging session. http://www.clinicaltrials.gov/ct2/show/NCT00978562, Accessed November 18, 2012

74. OHSU Knight Cancer Institute. MRI using ferumoxytol in patients with primary brain cancer or brain metastases from lung or breast cancer. http://clinicaltrials.gov/ct2/show/NCT00103038. Accessed November 18, 2012

75. OHSU Knight Cancer Institute. Magnetic resonance (MR) imaging study using ferumoxytol to assess early tumor response in patients with glioblastoma multiforme. http://clinicaltrials.gov/ show/NCT00660543. Accessed November 18, 2012

76. OHSU Knight Cancer Institute. Ferumoxytol and gadolinium magnetic resonance imaging (MRI) at $3 \mathrm{~T}$ and $7 \mathrm{~T}$ in patients with malignant brain tumors. http://clinicaltrials.gov/ct2/show/NCT00659126. Accessed November 18, 2012

77. OHSU Knight Cancer Institute. Assessing dynamic magnetic resonance (MR) imaging in patients with recurrent high grade glioma receiving chemotherapy (code 7228). http://clinicaltrials.gov/ct2/ show/NCT00769093. Accessed November 18, 2012

78. Oregon Health and Science University NIoNDaSN. MR, histologic and EM imaging of intravenous ferumoxytol in central nervous system (CNS) inflammation. http://clinicaltrials.gov/ct2/show/ NCT00659776. Accessed November 18, 2012 